\title{
Cancers attribuables à l'excès de poids au Canada en 2010
}

\section{Dianne Zakaria, Ph. D.; Amanda Shaw, M. Sc.}

Cet article a fait l'objet d'une évaluation par les pairs.

\section{Résumé}

Introduction : L'excès de poids (indice de masse corporelle [IMC] de 25,00 $\mathrm{kg} / \mathrm{m}^{2}$ ou plus) est un facteur de risque bien connu de diabète, d'hypertension et de maladie cardiovasculaire, mais on en sait moins sur son lien avec le cancer. Dans cette étude, nous avons utilisé le risque attribuable dans la population (RAP) pour estimer le fardeau des cancers attribuables à l'excès de poids chez les adultes canadiens (de 25 ans ou plus) en 2010 .

Méthodologie : Nous avons estimé les RAP en utilisant des estimations du risque relatif (RR) tirées du Continuous Update Project du World Cancer Research Fund International, des estimations du surpoids et de l'obésité fondées sur l'IMC tirées de l'Enquête sur la santé dans les collectivités canadiennes de 2000-2001 (surpoids : 25,00 à 29,99 kg/m²; obésité : $30,00 \mathrm{~kg} / \mathrm{m}^{2}$ et plus) et nous avons utilisé les nombres de cas de cancer figurant dans le Registre canadien du cancer. Les RAP ont été fondés sur des IMC corrigés pour tenir compte du biais associé à l'autodéclaration de la taille et du poids.

Résultats : Au Canada, en 2010, on peut attribuer environ 9645 cas de cancer à un excès de poids, ce qui représente $5,7 \%$ de tous les cas de cancer (hommes : 4,9\%; femmes : $6,5 \%$ ). En limitant l'analyse aux types de cancer associés à un IMC élevé, le RAP augmente à 14,9 \% (hommes : 17,5\%; femmes : 13,3\%). Les types de cancer pour lesquels le RAP était le plus élevé étaient l'adénocarcinome de l'œsophage $(42,2 \%)$, le cancer du rein $(25,4 \%)$, le cancer du cardia $(20,7 \%)$, le cancer du foie $(20,5 \%)$, le cancer du côlon $(20,5 \%)$ et le cancer de la vésicule biliaire $(20,2 \%)$ chez les hommes, et l'adénocarcinome de l'œsophage $(36,1 \%)$, le cancer de l'utérus $(35,2 \%)$, le cancer de la vésicule biliaire $(23,7 \%)$ et le cancer du rein $(23,0 \%)$ chez les femmes. Les types de cancer pour lesquels le nombre de cas attribuables était le plus élevé étaient le cancer du côlon (1 445), le cancer du rein (780) et le cancer de la prostate à un stade avancé (515) chez les hommes, et le cancer de l'utérus (1 825), le cancer du sein postménopausique (1 765) et le cancer du côlon (675) chez les femmes. Quels que soient le sexe et le type de cancer, les RAP étaient les plus élevés dans les Prairies (sauf en Alberta) et la région de l'Atlantique, et les plus faibles en Colombie-Britannique et au Québec.

Conclusion : Le fardeau du cancer attribuable à l'excès de poids est considérable et continuera de croître à court terme en raison de la hausse de la prévalence du surpoids et de l’obésité au Canada.

Mots-clés : risque attribuable dans la population, indice de masse corporelle, surpoids, obésité, cancer

\section{Introduction}

Le fardeau du cancer pèse lourd sur la population canadienne. À l'heure actuelle, le risque à vie de cancer est de $45 \%$ chez les hommes et de $42 \%$ chez les femmes ${ }^{1}$, et le cancer est la principale cause de décès au Canada, étant à l'origine de $30 \%$ des décès en $2012^{2}$. La compréhension des principaux facteurs associés au fardeau du cancer constitue donc une priorité en santé. On peut utiliser le risque attribuable dans la population (RAP) pour quantifier les répercussions de divers facteurs sur l'apparition du cancer dans une population, ce qui en fait un outil important permettant de prioriser les stratégies de lutte contre le cancer. Reposant sur le principe
Points saillants

- Environ 9645 cas de cancer, soit $5,7 \%$ de l'ensemble des cancers diagnostiqués chez les adultes canadiens (de 25 ans ou plus), étaient attribuables à un excès de poids en 2010.

- Les cancers pour lesquels la proportion des cas attribuables à un excès de poids était la plus élevée étaient l'adénocarcinome de l'œsophage, le cancer du rein, le cancer du cardia, le cancer du foie, le cancer du côlon et le cancer de la vésicule biliaire chez les hommes et l'adénocarcinome de l'œsophage, le cancer de l'utérus, le cancer de la vésicule biliaire et le cancer du rein chez les femmes.

- Les cancers pour lesquels le nombre de cas attribuables à un excès de poids était le plus élevé étaient le cancer du côlon, le cancer du rein et le cancer de la prostate à un stade avancé chez les hommes et le cancer de l'utérus, le cancer du sein postménopausique et le cancer du côlon chez les femmes.

- La proportion la plus élevée de cas de cancer attribuables à un excès de poids était dans les Prairies (sauf en Alberta) et la région de l'Atlantique, et la plus faible en ColombieBritannique et au Québec.

qu'il existe une relation de cause à effet entre un facteur donné et le cancer, le RAP permet d'estimer la proportion de cas de cancer évitables dans une population en éliminant le facteur en question ${ }^{3,4}$. Dans une récente étude menée au Royaume-Uni faisant appel au RAP pour estimer la proportion de cas de cancer attribuables au mode de vie et aux facteurs de risques environnementaux, le

Rattachement des auteures :

Agence de la santé publique du Canada, Ottawa (Ontario), Canada

Correspondance : Dianne Zakaria, Agence de la santé publique du Canada, 785, avenue Carling, pièce 611B1, Ottawa (Ontario) K1A 0K9; tél. : 613-240-1713; courriel : Dianne.Zakaria@phac-aspc.gc.ca 
tabagisme, l'alimentation et l'excès de poids ont été décrits comme les trois principaux facteurs de risque, correspondant à respectivement $19,4 \%, 9,2 \%$ et $5,5 \%$ de tous les cancers ${ }^{5}$.

Si l'excès de poids est un facteur de risque bien connu de diabète, d'hypertension et de maladie cardiovasculaire, on en sait moins sur son lien avec le cancer ${ }^{6}$. En 2002, le Centre international de recherche sur le cancer a conclu que l'excès de poids était associé à un risque accru de cancer du côlon, de cancer du sein (postménopausique), de cancer de l'endomètre, de cancer du rein et de cancer de l'œsophage (adénocarcinome) $^{6}$. Depuis, dans le cadre de recensions systématiques, d'autres cancers ont aussi été identifiés ${ }^{7-10}$. Les mécanismes potentiels de cancérogenèse cités dans ces articles sont des altérations hormonales et métaboliques, un taux élevé de stress oxydatif, une stimulation de la réponse inflammatoire de l'organisme et une accentuation du phénomène de reflux gastroœsophagien causée par les effets mécaniques directs d'une obésité centrale $e^{6,7,11}$.

Dans plusieurs études relativement récentes, on s'est intéressé à la proportion de cas de cancer attribuables à un excès de poids au Canada ${ }^{12,13}$, et plus particulièrement en Ontario ${ }^{14}$. Depuis, une quantité considérable de données a permis de déterminer qu'il y avait une relation de cause à effet entre l'excès de poids et d'autres types de cancer. De plus, aucun des travaux de recherche menés antérieurement n'a offert d'examen exhaustif des RAP par région au Canada. Étant donné qu'il est de plus en plus clair que le poids est un facteur de risque de cancer et étant donné que le nombre de personnes en surpoids est en augmentation au sein de la population canadienne ${ }^{15}$, nous avons besoin de davantage d'estimations exhaustives et à jour de la proportion de cancers attribuables à l'excès de poids pour orienter les stratégies de lutte contre le cancer. L'objectif principal de notre étude est ainsi d'estimer la proportion et le nombre de nouveaux cas de cancer attribuables à un excès de poids chez les adultes canadiens de 25 ans ou plus en 2010.

\section{Méthodologie}

\section{Prévalence de l'excès de poids dans la population canadienne}

Nous avons utilisé l'indice de masse corporelle (IMC), une unité de mesure fréquemment utilisée et associée à des seuils pour l'excès de poids ${ }^{16}$ (soit un IMC supérieur ou égal à $25,00 \mathrm{~kg} / \mathrm{m}^{2}$; tableau 1 ), pour quantifier la prévalence du surpoids et de l'obésité chez les Canadiens. Nous avons utilisé les données autodéclarées sur la taille et le poids tirées de l'Enquête sur la santé dans les collectivités canadiennes (ESCC) ${ }^{17}$ pour calculer l'IMC des sujets (soit leur poids en kilogrammes divisé par le carré de leur taille en mètres). L’ESCC, une enquête menée auprès de la population depuis l'an 2000, est conçue pour fournir des estimations fiables à l'échelle des régions sanitaires et des provinces pour les personnes de 12 ans ou plus, avec quelques exclusions représentant moins de $3 \%$ de la population canadienne. Les travaux de recherche menés au Canada ont permis de déterminer que l'IMC fondé sur des données autodéclarées est inférieur à la réalité, car les gens ont tendance à fournir une mesure de la taille supérieure et une mesure du poids inférieure à la réalité ${ }^{18}$. Par conséquent, dans notre étude, nous avons corrigé les IMC au moyen de formules élaborées à partir d'un sous-échantillon de répondants à l'ESCC ayant accepté, outre de fournir une autodéclaration de leur taille et de leur poids, de se faire mesurer et peser (équations 1 et 2) ) $^{19,20}$.

Pour estimer la proportion de cas de cancer attribuables à un excès de poids en 2010, nous avons utilisé les données de 2000 et 2001 sur la prévalence de l'excès de poids, de façon à laisser une période de latence d'au moins 10 ans entre le moment de l'exposition (excès de poids) et l'apparition de la maladie (cancer). Ainsi, par exemple, la proportion de cas de cancer attribuables à un excès de poids chez les adultes de 45 à 54 ans en 2010 est fondée sur la prévalence du surpoids et de l'obésité chez les adultes de 35 à 44 ans en 2000 et 2001 . Une période de latence de 10 ans était le plus long intervalle utilisable en se fondant sur l'ESCC. De plus, cette période concorde avec ce qui s'est fait dans des travaux de recherche similaires ${ }^{12,21,22}$ et avec l'intervalle de la moyenne géométrique de la durée du suivi dans une méta-analyse exhaustive portant sur l'association entre le poids et divers types de cancer ${ }^{8}$. Pour reproduire le plan d'échantillonnage de l'ESCC, nous
TABLEAU 1

\section{Catégories d'indice de masse corporelle}

\begin{tabular}{lc}
\multicolumn{1}{c}{ Catégorie } & \multicolumn{1}{c}{ Seuil } \\
\hline $\begin{array}{l}\text { Insuffisance } \\
\text { pondérale }\end{array}$ & Moins de $18,50 \mathrm{~kg} / \mathrm{m}^{2}$ \\
$\begin{array}{l}\text { Éventail de poids } \\
\text { normal }\end{array}$ & 18,50 à $24,99 \mathrm{~kg} / \mathrm{m}^{2}$ \\
$\begin{array}{l}\text { Surpoids } \\
\text { Obésité }\end{array}$ & $25,00 \mathrm{à} 29,99 \mathrm{~kg} / \mathrm{m}^{2}$ \\
\hline
\end{tabular}

Remarque : Catégories conformes aux définitions de l'OMS présentées dans le document Obésité : prévention et prise en chargée de l'épidémie mondiale ${ }^{16}$.

avons pondéré toutes les estimations et nous avons obtenu les estimations correspondantes de la variance en nous servant de la technique dite de "répétition compensée », en utilisant les poids de rééchantillonnage fournis. Le taux de réponse à l'ESCC en 2000 et 2001 était de 84,7\% à l'échelle nationale ${ }^{17}$.

\section{Associations entre l'excès de poids et le cancer}

Les cancers pour lesquels nous possédons des preuves solides de l'existence d'une relation de cause à effet avec un IMC élevé ont été déterminés à partir du Continuous Update Project (CUP) du World Cancer Research Fund (WCRF) International, un programme permanent qui analyse les travaux de recherche menés à l'échelle internationale sur les façons dont l'alimentation, la nutrition, l'activité physique et le poids influent sur le risque et la survie associés au cancer ${ }^{23}$. Le CUP effectue des examens systématiques approfondis et des méta-analyses portant essentiellement sur des essais contrôlés randomisés, des études de cohorte et des études cas-témoins nichées. Les méta-analyses sont fondées sur les estimations d'associations les plus ajustées en fonction des variables de confusion et pour lesquelles les données sont congruentes à une relation dose-effet. Ces examens sont évalués par un comité d'experts indépendants qui fournissent des conclusions à propos de la qualité des preuves appuyant l'existence de ces relations. Les données sont jugées suffisamment probantes si elles permettent de justifier de façon générale les recommandations destinées à réduire l'incidence du 
cancer. Elles sont jugées probantes si elles remplissent les conditions suivantes : données tirées d'au moins deux études de cohorte indépendantes ou d'au moins cinq études cas-témoins; absence d'hétérogénéité notable inexpliquée; études de qualité excluant la possibilité d'erreurs aléatoires ou systématiques et enfin plausibilité sur le plan biologique. Les autres critères sont les suivants : données tirées de plus d'un type d'études; présence d'une relation doseréponse et enfin données probantes et plausibles tirées d'études menées chez des humains ou des animaux selon lesquelles les types d'expositions jugés usuels pour les humains peuvent mener à l'apparition d'un cancer ${ }^{24}$.

Selon le CUP du WCRF International, il existe des données probantes prouvant l'existence d'une relation de cause à effet entre un IMC élevé et 12 cancers (tableau 2). Les estimations du risque relatif (RR) spécifique au sexe extraites pour notre étude sont fondées sur des études de cohorte portant sur les nouveaux cas de cancer. Nous avons converti les estimations du RR associées à une augmentation de cinq unités de l'IMC en des estimations du RR associées à une augmentation d'une unité de l'IMC, en supposant une relation linéaire entre le logarithme naturel du RR et l'IMC. Chez les hommes, le RR de cancer associé à une augmentation d'une unité de l'IMC allait d'une valeur minimale de 1,02 (pour le cancer du pancréas, le cancer du rectum et le cancer de la prostate à un stade avancé) à une valeur maximale de 1,09 (pour l'adénocarcinome de l'œsophage). Chez les femmes, le risque accru allait d'une valeur minimale de 1,01 (pour le cancer du rectum et le cancer de l'ovaire) à une valeur maximale de 1,08 (pour l'adénocarcinome de l'œsophage et le cancer de l'utérus).

\section{Nombre de nouveaux cas de cancer au Canada en 2010}

Nous avons obtenu le nombre de nouveaux cas de cancer pour chaque province et territoire (à l'exception du Québec) pour l'année la plus récente associée à des données complètes à l'échelle nationale (2010) en consultant le Registre canadien du cancer (RCC) (fichier de données fondé sur le document International Rules for Multiple Primary Cancers ${ }^{35}$, publié en septembre 2012). Dans le cas du Québec, nous avons téléchargé les données sur le nombre

TABLEAU 2

Risques relatifs de 12 types de cancer associés à une augmentation d'une unité de l'indice de masse corporelle, en fonction du sexe

\begin{tabular}{lcc} 
& \multicolumn{2}{c}{ Risque relatif $(\mathrm{IC}$ à $95 \%)$} \\
\hline Adénocarcinome de l'œsophagé $^{24}$ & Hommes & Femmes \\
Cancer du cardia $^{25, \mathrm{~b}}$ & $1,09(1,07$ à 1,12$)$ & $1,08(1,05$ à 1,11$)$ \\
Cancer du foie $^{26}$ & $1,04(1,01$ à 1,07$)$ & $1,04(1,01$ à 1,07$)$ \\
Cancer de la vésicule biliaire $^{27}$ & $1,04(1,00$ à 1,08$)$ & $1,04(1,02$ à 1,06$)$ \\
Cancer du pancréas $^{28}$ & $1,04(1,02$ à 1,06$)$ & $1,05(1,01$ à 1,08$)$ \\
Cancer du côlon $^{29}$ & $1,02(1,01$ à 1,04$)$ & $1,02(1,01$ à 1,03$)$ \\
Cancer du rectum $^{29}$ & $1,04(1,03$ à 1,05$)$ & $1,02(1,01$ à 1,03$)$ \\
Cancer du rein $^{30}$ & $1,02(1,01$ à 1,02$)$ & $1,01(1,00$ à 1,02$)$ \\
Cancer de la prostate à un stade $_{\text {avancé }^{31}}$ & $1,05(1,04$ à 1,06$)$ & $1,05(1,04$ à 1,06$)$ \\
Cancer du sein postménopausique $^{32}$ & $1,02(1,01$ à 1,02$)$ & S.0. \\
Cancer de l'utérus $^{33}$ & S.0. & $1,02(1,02$ à 1,03$)$ \\
Cancer de l'ovaire $^{34}$ & S.O. & $1,08(1,07$ à 1,10$)$ \\
\hline
\end{tabular}

Abréviations : IC, intervalle de confiance; S.O., sans objet.

Remarque : Les estimations du risque relatif (RR) associées à une augmentation de cinq unités de l'indice de masse corporelle (IMC) ont été converties en des estimations du RR associées à une augmentation d'une unité de l'IMC, sur la base d'une relation linéaire entre le logarithme naturel du RR et l'IMC.

a Types de cancer selon le CUP du World Cancer Research Fund International23.

${ }^{\mathrm{b}}$ Les estimations concernent à la fois les hommes et les femmes, car les estimations spécifiques au sexe étaient fondées sur un nombre insuffisant d'études.

de cas survenus en 2010 à partir du site Internet de Statistique Canada ${ }^{36}$.

Les définitions de cancer ont été choisies et classées en fonction des méta-analyses quantifiant leur association avec l'IMC (tableau 3). Étant donné que l'information sur la stadification n'était pas complète pour tous les cas de cancer de la prostate, en particulier dans certaines provinces, nous avons obtenu une estimation de la proportion des cas de cancer de la prostate de stade III et de stade IV en fonction des groupes d'âge en utilisant l'information sur tous les diagnostics de cancer de la prostate avec stadification posés dans l'ensemble du Canada (à l'exclusion du Québec) en 2010.

Pour le Québec, nous avons dû corriger le nombre de cas de certains types de cancer pour lesquels il n'y avait aucunes données directement accessibles par l'intermédiaire $\mathrm{du}$ site Internet de Statistique Canada. Nous avons pour cela estimé le nombre de cas d'adénocarcinome de l'œsophage, de cancer du cardia et de cancer du foie (y compris des voies biliaires intrahépatiques) au Québec en utilisant l'information disponible pour l'ensemble des provinces et des territoires, à l'exclusion du Québec. Par exemple, pour estimer la proportion, au Québec, de cas de cancer de l'œsophage correspondant aux adénocarcinomes, nous avons utilisé les proportions spécifiques au sexe et spécifiques à l'âge pour l'ensemble du Canada à l'exception du Québec pour 2010. Pour des raisons de confidentialité, les nombres de cas présentés ont été arrondis au multiple de 5 supérieur ou inférieur au moyen d'un modèle d'arrondissement aléatoire sans biais et les nombres de cas se terminant déjà par un « 0 » ou un « 5 » n'ont pas été arrondis.

\section{Risque attribuable dans la population (RAP)}

Nous avons estimé les RAP par cancers, régions, sexes et groupes d'âge en utilisant l'équation $3^{4}$.

Nous avons quantifié le RR associé à une catégorie d'IMC par rapport à la valeur de $21 \mathrm{~kg} / \mathrm{m}^{2}$, qui constitue une valeur optimale reconnue ${ }^{40}$, en utilisant l'équation 4 . 
TABLEAU 3

Définition des cancers associés à un excès de poids ${ }^{\mathrm{a}}$

\begin{tabular}{lll}
\multicolumn{1}{c}{ Cancer } & \multicolumn{1}{c}{ Topographie } & \multicolumn{1}{c}{ Histologie } \\
\hline Adénocarcinome de l'œesophage ${ }^{\mathrm{b}}$ & C15.0-C15.9 & $\begin{array}{l}\text { 8050, 8140-8147, 8160-8162, 8180-8221, 8250-8507, 8514, } \\
\text { 8520-8551, 8560, 8570-8574, 8576, 8940-8941 }\end{array}$ \\
Cancer du cardia & C16.0 & à l'exclusion de 9050-9055, 9140, 9590-9992 \\
Cancer du foie & C22.0, C22.1 & à l'exclusion de 9050-9055, 9140, 9590-9992 \\
Cancer de la vésicule biliaire & C23.9 & à l'exclusion de 9050-9055, 9140, 9590-9992 \\
Cancer du pancréas & C25.0-C25.9 & à l'exclusion de 9050-9055, 9140, 9590-9992 \\
Cancer du côlon & C18.0-C18.9, C26.0 & à l'exclusion de 9050-9055, 9140, 9590-9992 \\
Cancer du rectum & C19.9, C20.9 & à l'exclusion de 9050-9055, 9140, 9590-9992 \\
Cancer du rein & C64.9, C65.9 & à l'exclusion de 9050-9055, 9140, 9590-9992 \\
Cancer de la prostate (stade avancé) & C61.9 àn l'exclusion de 9050-9055, 9140, 9590-9992 \\
Cancer du sein (postménopausique) & C50.0-C50.9 & à l'exclusion de 9050-9055, 9140, 9590-9992 \\
Cancer de l'utérus & C54.0-C54.9, C55.9 & à l'exclusion de 9050-9055, 9140, 9590-9992 \\
Cancer de l'ovaire & C56.9 & à l'exclusion de 9050-9055, 9140, 9590-9992 \\
\hline
\end{tabular}

Abréviation : AJCC, American Joint Committee on Cancer.

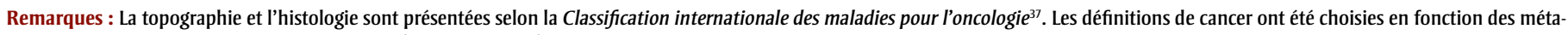

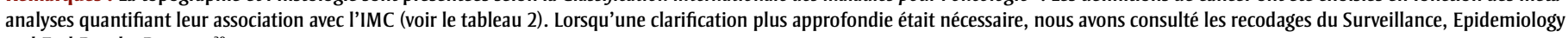
and End Results Program ${ }^{38}$.

${ }^{a}$ Excès de poids : IMC $\geq 25,00 \mathrm{~kg} / \mathrm{m}^{2}$.

${ }^{\text {b }}$ Adénocarcinome de l'œsophage : selon la définition de Howlader et collab ${ }^{39}$.

Nous avons supposé qu'il n'y avait aucun risque pour les IMC inférieurs à $25 \mathrm{~kg} / \mathrm{m}^{2}$.

Le produit entre le RAP spécifique au cancer, à la région, au sexe et au groupe d'âge

et le nombre de nouveaux cas de cancer nous a permis d'obtenir le nombre de cas de cancer attribuables à un excès de poids. Nous avons ensuite effectué des sommes dans les strates pertinentes (p. ex. type de

\section{$\mathrm{RAP}=\left[\mathrm{P}_{\mathrm{s}}\left(\mathrm{RR}_{\mathrm{s}}-1\right)+\mathrm{P}_{\mathrm{OB}}\left(\mathrm{RR}_{\mathrm{OB}}-1\right)\right]$ \\ $\left[1+\left(P_{S}\left(R R_{S}-1\right)+P_{O B}\left(R R_{O B}-1\right)\right)\right]$ \\ où : \\ $P_{\mathrm{S}}=$ proportion de personnes considérées comme étant en surpoids 10 ans avant 2010 \\ $\mathrm{P}_{\mathrm{ов}}=$ proportion de personnes considérées comme étant obèses 10 ans avant 2010 \\ $\mathrm{RR}_{\mathrm{S}}=$ risque relatif associé à l'IMC médian de la catégorie « surpoids » par rapport à $21 \mathrm{~kg} / \mathrm{m}^{2}$, en supposant une relation log-linéaire entre le $R R$ et l'IMC \\ $\mathrm{RR}_{\mathrm{OB}}=$ risque relatif associé à l'IMC médian de la catégorie « obèse » par rapport à $21 \mathrm{~kg} / \mathrm{m}^{2}$, en supposant une relation log-linéaire entre le $R R$ et l'IMC}

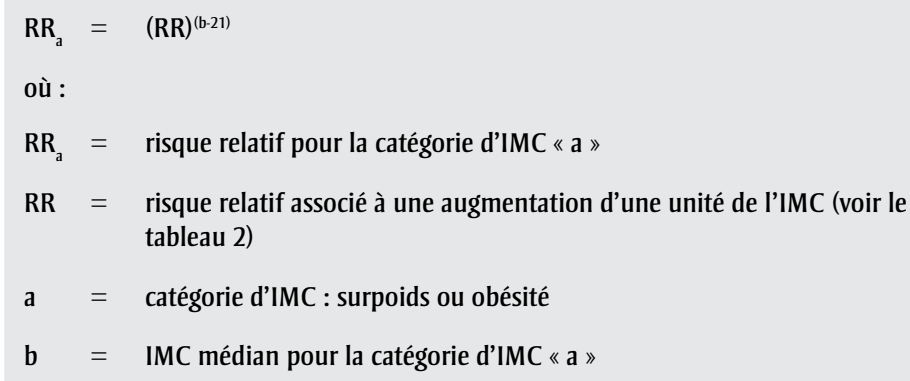

(Équation 3)

(Équation 4) cancer, région, sexe, âge) pour obtenir les RAP et le nombre de cas attribuables pour chaque sous-groupe à l'étude. Étant donné que la prévalence de l'excès de poids varie selon la région et le sexe et que la force de l'association entre l'IMC et le cancer est susceptible de varier en fonction du sexe, nous avons estimé la proportion et le nombre de nouveaux cas de cancer attribuables à un excès de poids en fonction de la province et du sexe. En ce qui concerne les territoires, nous les avons considérés comme une seule et même région et avons regroupé les données correspondantes. Afin de permettre une période de latence de 10 ans et d'obtenir des estimations plus stables, nous avons défini les groupes d'âge de la façon suivante : de 25 à 34 ans, de 35 à 44 ans, de 45 à 54 ans, de 55 à 64 ans, de 65 à 74 ans, de 75 à 84 ans, et 85 ans ou plus. Nous n'avons pas estimé les RAP et le nombre de cas attribuables pour le groupe des 15 à 24 ans, car l'ESCC ne permettait pas d'obtenir de données sur la prévalence du surpoids et de l'obésité chez les jeunes de 5 à 14 ans en 2000 et 2001. Afin de tenir compte de l'incertitude relative à l'ampleur de la relation entre l'excès de poids et le risque de cancer, nous avons estimé une plage plausible de RAP et de nombres de cas attribuables en utilisant les intervalles de confiance à $95 \%$ associés au RR du tableau 2. 


\section{Résultats}

\section{Prévalence de l'excès de poids}

Notre étude n'avait pas pour objectif primaire d'effectuer un examen détaillé de la prévalence de l'excès de poids, mais plutôt d'estimer la prévalence de l'excès de poids en fonction de la région, du sexe et du groupe d'âge afin d'estimer les RAP. Il convient néanmoins d'apporter quelques précisions au sujet de la prévalence de l'excès de poids étant donné qu'elle constitue l'une des principales composantes du RAP. Premièrement, mentionnons que pour l'ensemble du pays, en 2000 et 2001, les hommes étaient plus susceptibles que les femmes d'être en surpoids, mais que la proportion de personnes considérées comme obèses était similaire chez les deux sexes (figure 1). Deuxièmement, précisons que la proportion d'adultes considérés comme étant en surpoids variait moins d'une région à l'autre que la proportion d'adultes considérés comme obèses. Ainsi, la prévalence d'adultes obèses la plus faible se situait en Colombie-Britannique et au Québec, et la plus élevée en Saskatchewan, au Manitoba, dans la région de l'Atlantique et dans les territoires.

\section{Cancers attribuables à l'excès de poids}

Les RAP, les nombres de cas attribuables et la plage de valeurs plausibles sont présentés en fonction du type de cancer, du sexe et de la région dans le tableau 4. Environ $5,7 \%$ de l'ensemble des cas de cancer diagnostiqués chez des adultes canadiens en 2010, soit 9645 cas, étaient attribuables à un excès de poids. En tenant compte de l'incertitude associée aux estimations du $\mathrm{RR}$, la plage de valeurs plausibles pour le RAP se situe entre $4,1 \%$ et $7,6 \%$, et le nombre de cas de cancer attribuables à un excès de poids entre 6980 et 12845 . Le RAP pour tous les types de cancer confondus était légèrement plus élevé chez les femmes que chez les hommes $(6,5 \%$ contre $4,9 \%$ ), en raison de cancers spécifiquement féminins associés à un excès de poids (cancer du sein postménopausique et cancer de l'utérus). Si on limite l'analyse aux types de cancer associés à un excès de poids (tableau 2), le RAP est plus élevé chez les hommes que chez les femmes $(17,5 \%$ contre $13,3 \%)$, parce que le RR associé à certains des types de cancer les plus courants (p. ex. le cancer du côlon et le cancer du rectum) s'est révélé plus élevé chez les hommes que chez les femmes et que les hommes étaient plus susceptibles que les femmes d'être en surpoids en 2000 et 2001 .

Si la proportion de l'ensemble des cas de cancer attribuables à un excès de poids peut sembler modeste, les effets d'un excès de poids sont, pour certains types de cancer, considérables. Par exemple, chez les hommes, on peut attribuer environ 42,2\% des cas d'adénocarcinome de l'œsophage, $25,4 \%$ des cas de cancer du rein et environ $20 \%$ des cas de cancer du cardia, de cancer du foie, de cancer de la vésicule biliaire et de cancer du côlon à un excès de poids. Chez les femmes, on peut attribuer 36,1 \% des cas d'adénocarcinome de l'œsophage, 35,2 \% des cas de cancer de l'utérus et près de 1 cas sur 4 de cancer du rein ou de cancer de la vésicule biliaire à un excès de poids. Quels que soient le type de cancer et le sexe, les RAP les plus faibles se situaient en Colombie-Britannique et au Québec, et les plus élevés dans les Prairies (sauf en Alberta) et dans la région de l'Atlantique, suivant grosso modo la prévalence de l'excès de poids dans ces régions en 2000 et en 2001.

Enfin, il convient de faire la distinction entre le RAP et le nombre de cas attribuables. Les cancers pour lesquels les cas attribuables à un excès de poids sont nombreux ne sont pas nécessairement ceux pour lesquels les RAP sont les plus élevés. Chez les hommes, le cancer du côlon arrive au quatrième rang du point de vue du RAP, mais au premier rang du point de vue du nombre de cas attribuables, ce qui correspond à environ le tiers de

FIGURE 1

Pourcentage de Canadiens de 15 ans ou plus considérés comme étant en surpoids ou obèses, en fonction du sexe, en 2000-2001

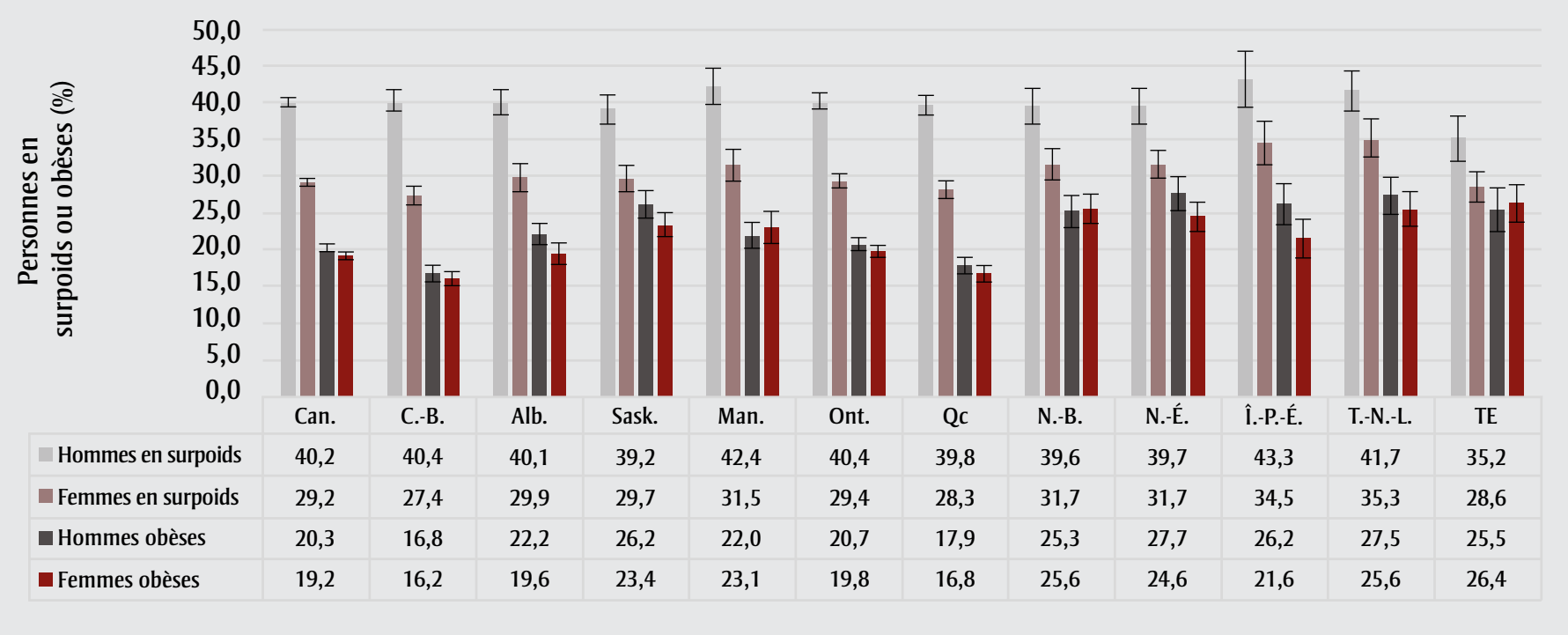

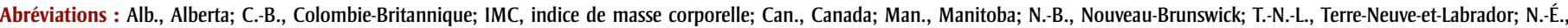
Nouvelle-Écosse; Ont., Ontario; Î.-P.-É., Île-du-Prince-Édouard; Qc, Québec; Sask., Saskatchewan; TE, les trois territoires canadiens réunis.

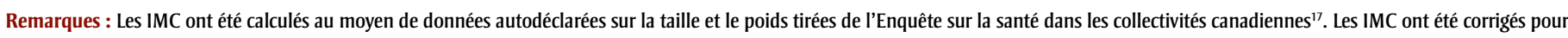

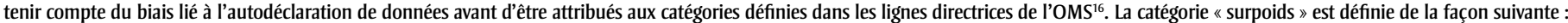
$25,00 \mathrm{~kg} / \mathrm{m}^{2} \leq \mathrm{IMC} \leq 29,99 \mathrm{~kg} / \mathrm{m}^{2}$. La catégorie « obèse » est définie de la façon suivante : IMC $\geq 30,00 \mathrm{~kg} / \mathrm{m}^{2}$. Les barres représentent les intervalles de confiance à $95 \%$. 


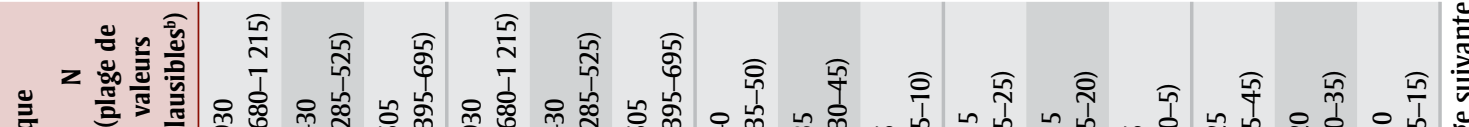

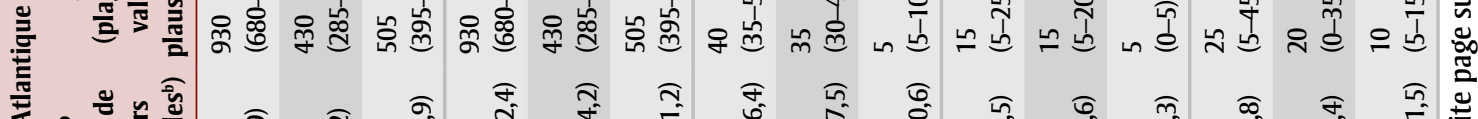

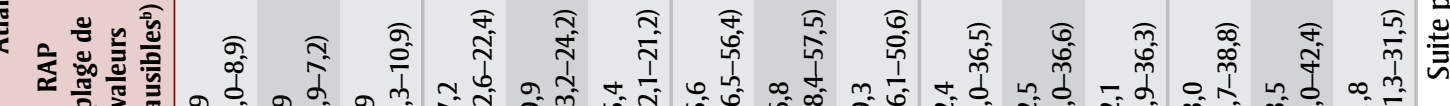

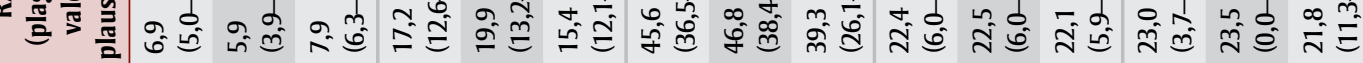

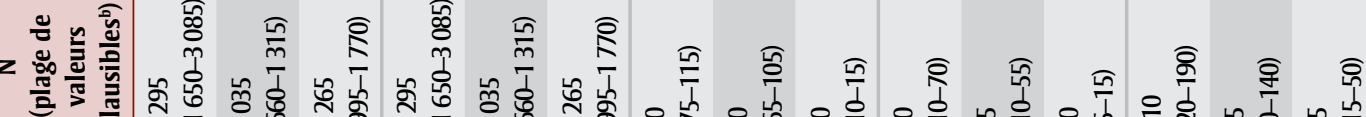

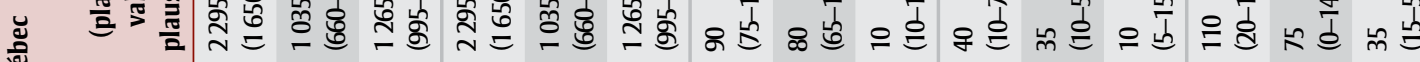

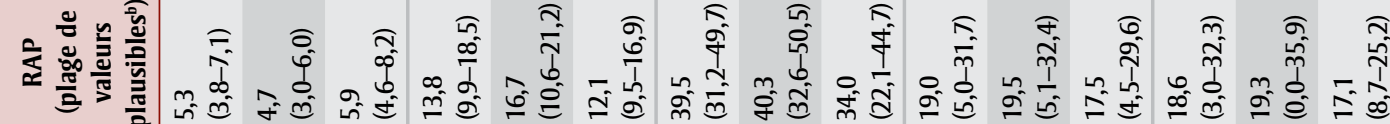

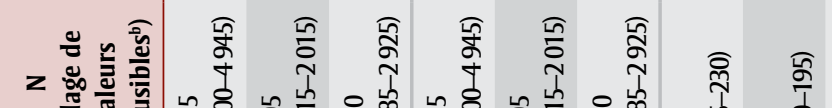

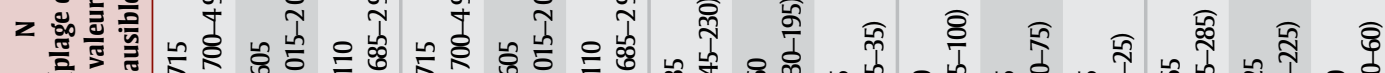

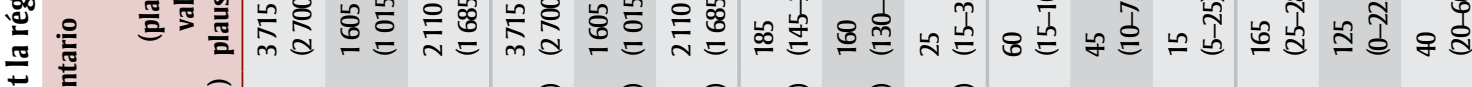
б

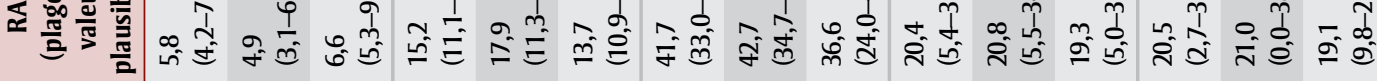

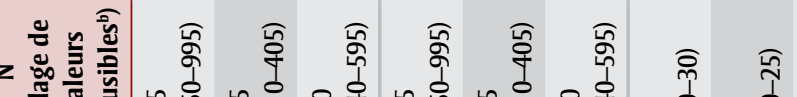

z

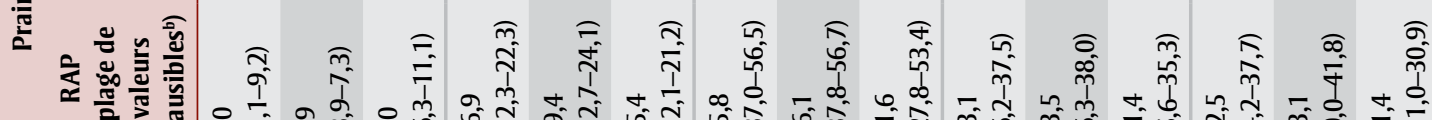

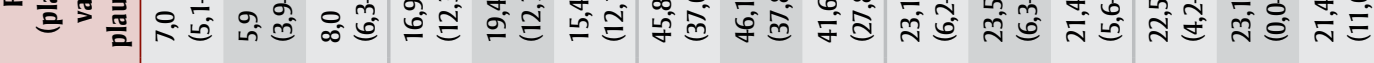

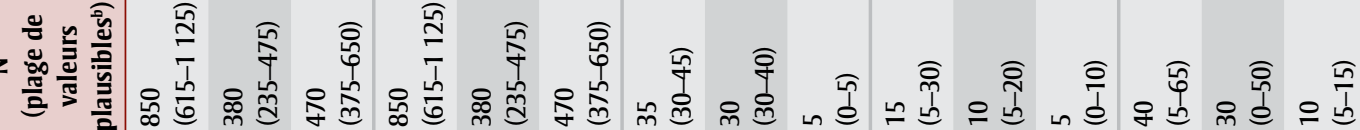

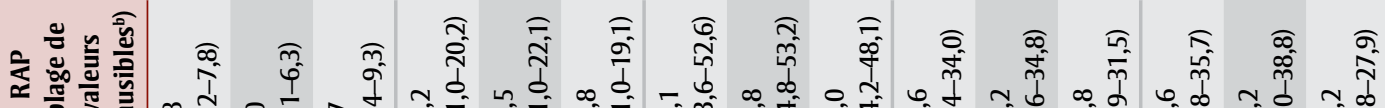

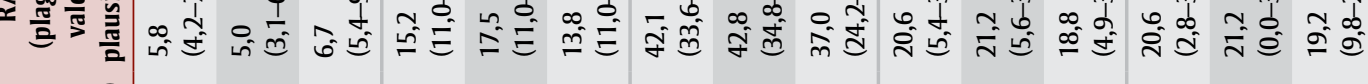

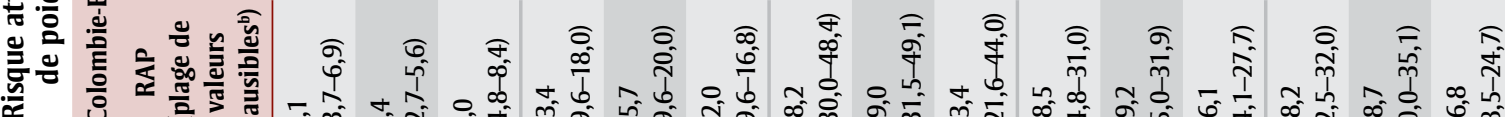

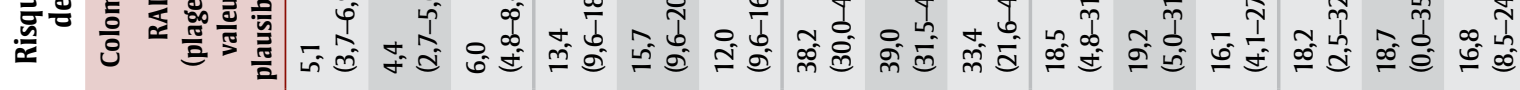

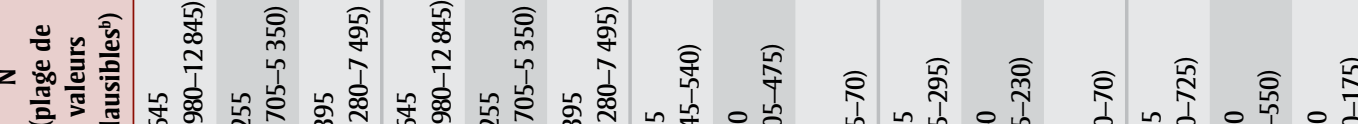

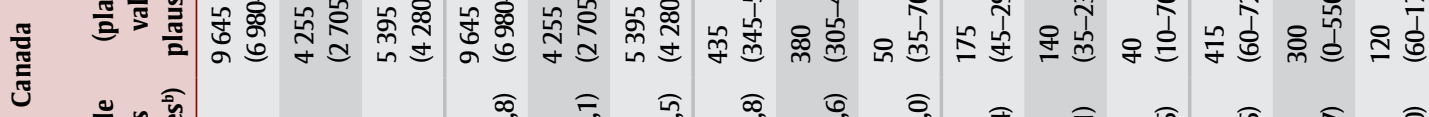

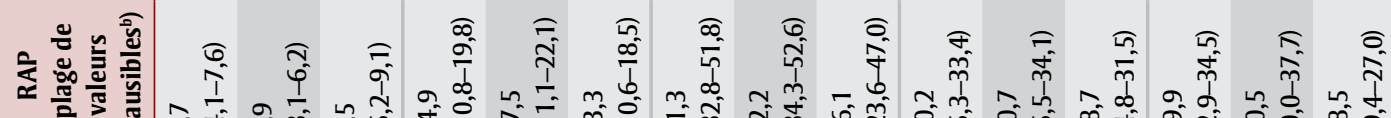

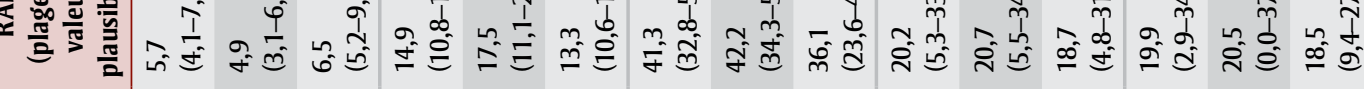

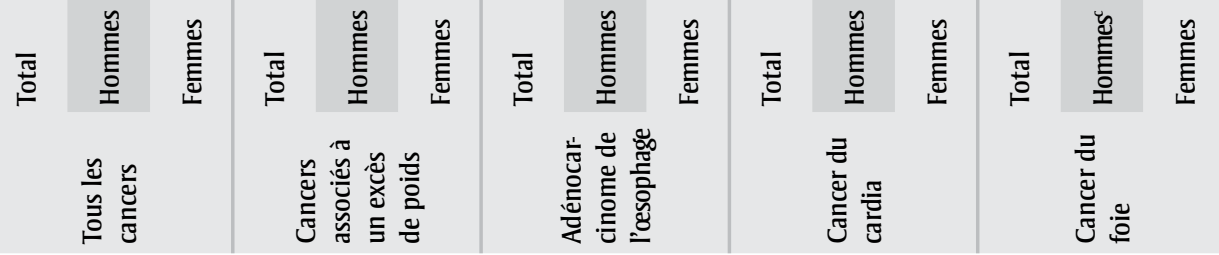




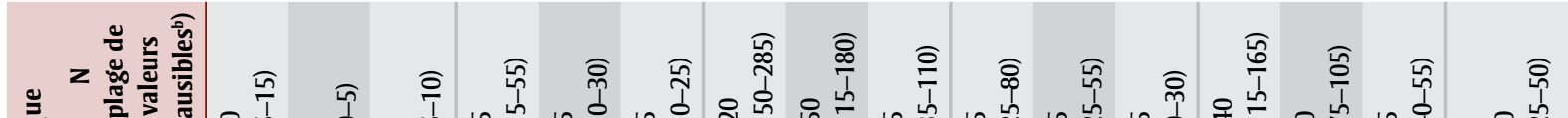

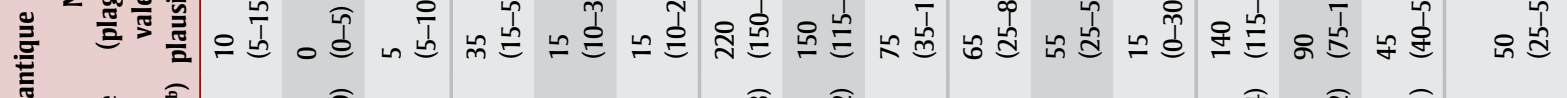
एँ

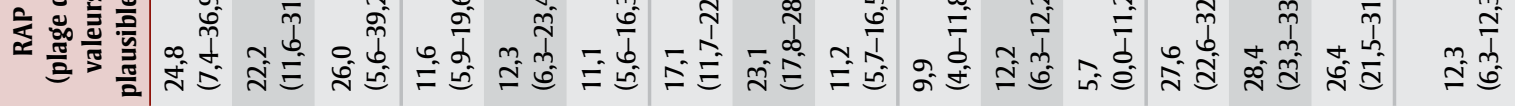

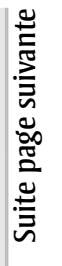
2 品 言

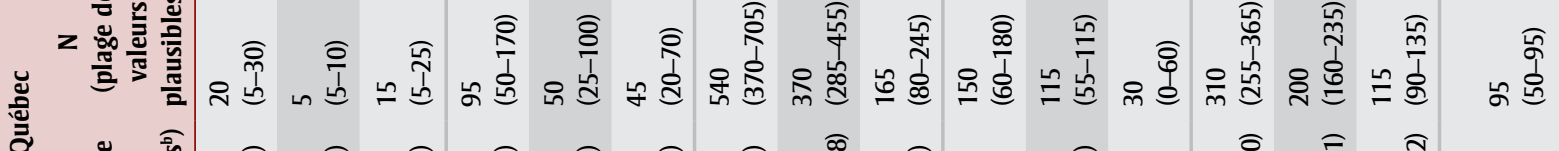

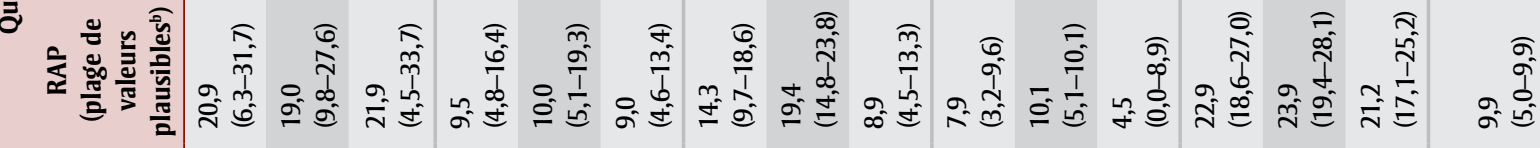

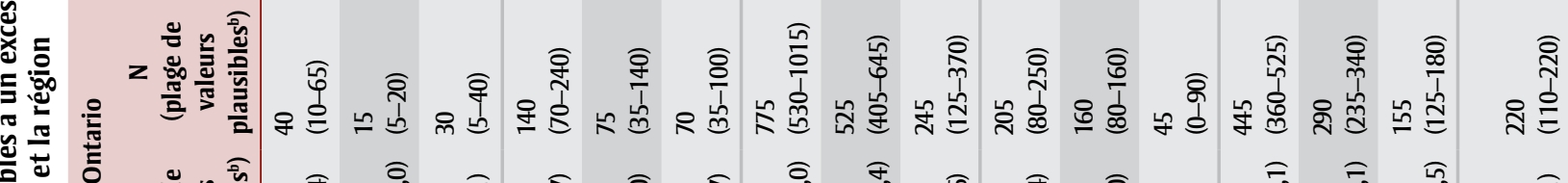

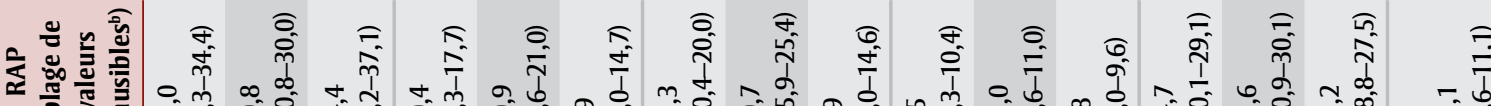

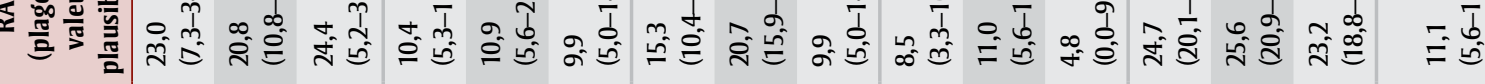

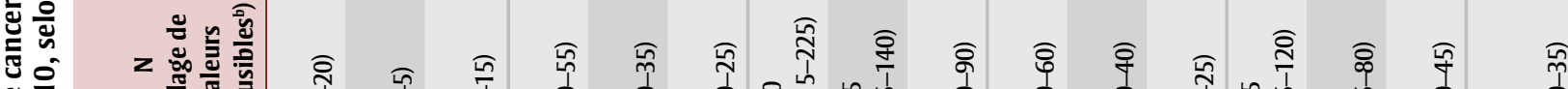

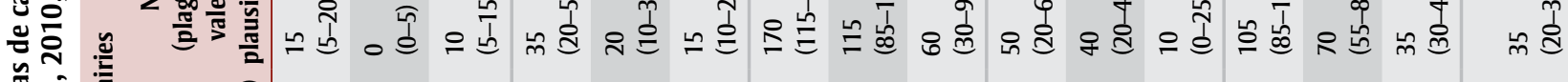
递室 ¿

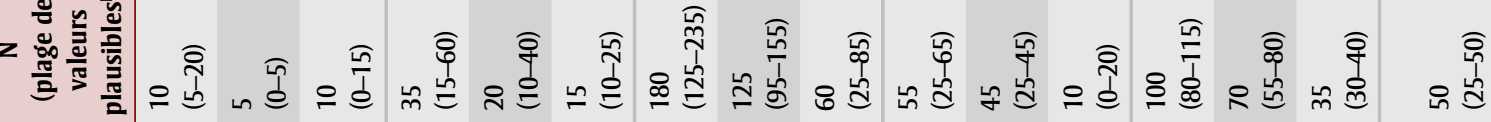

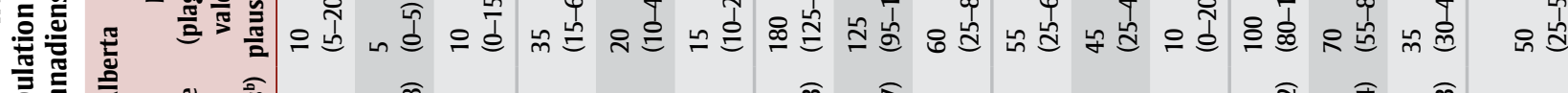

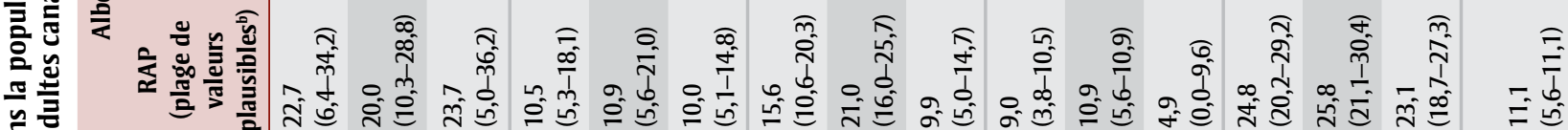
焉

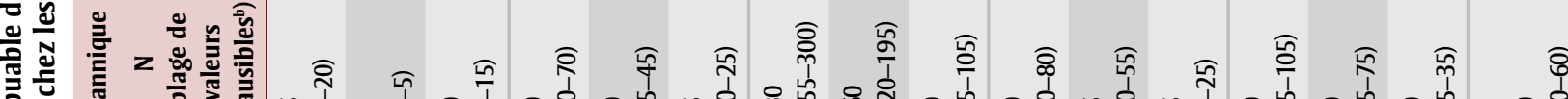

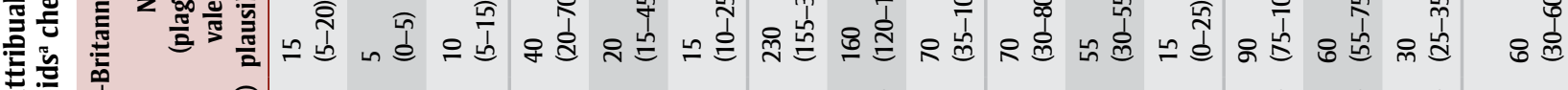

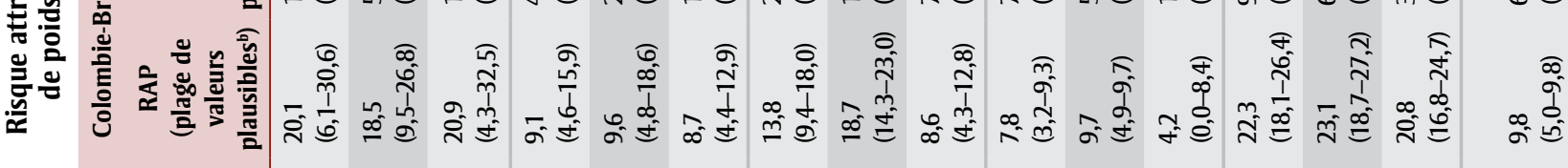

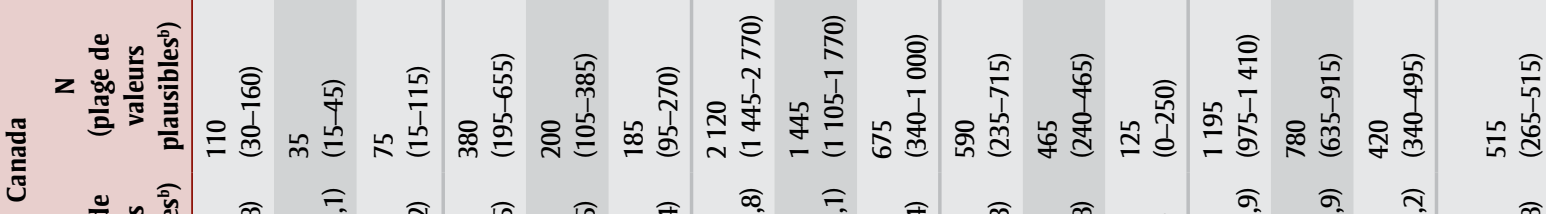

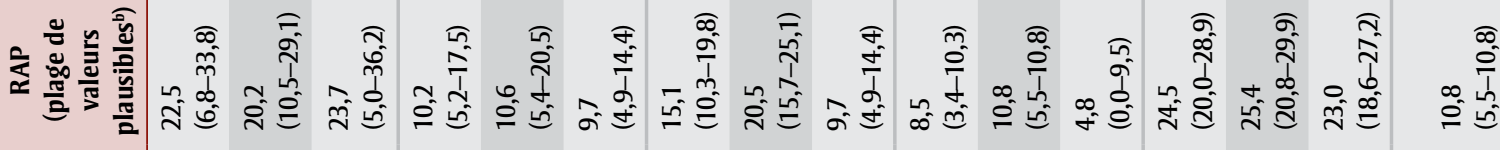

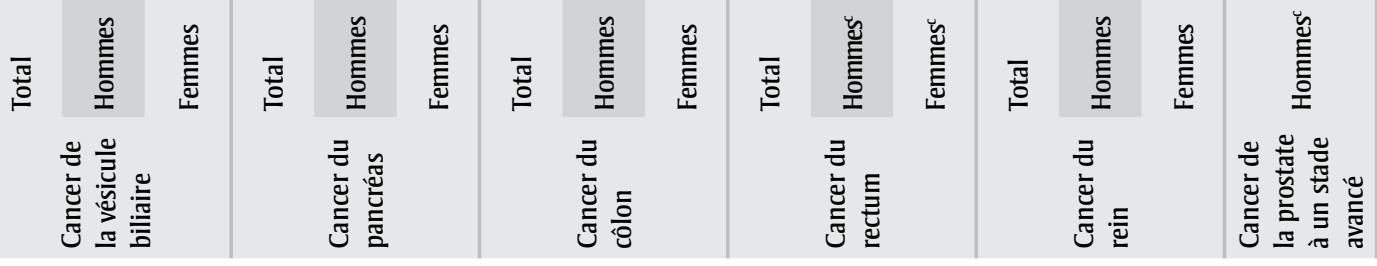




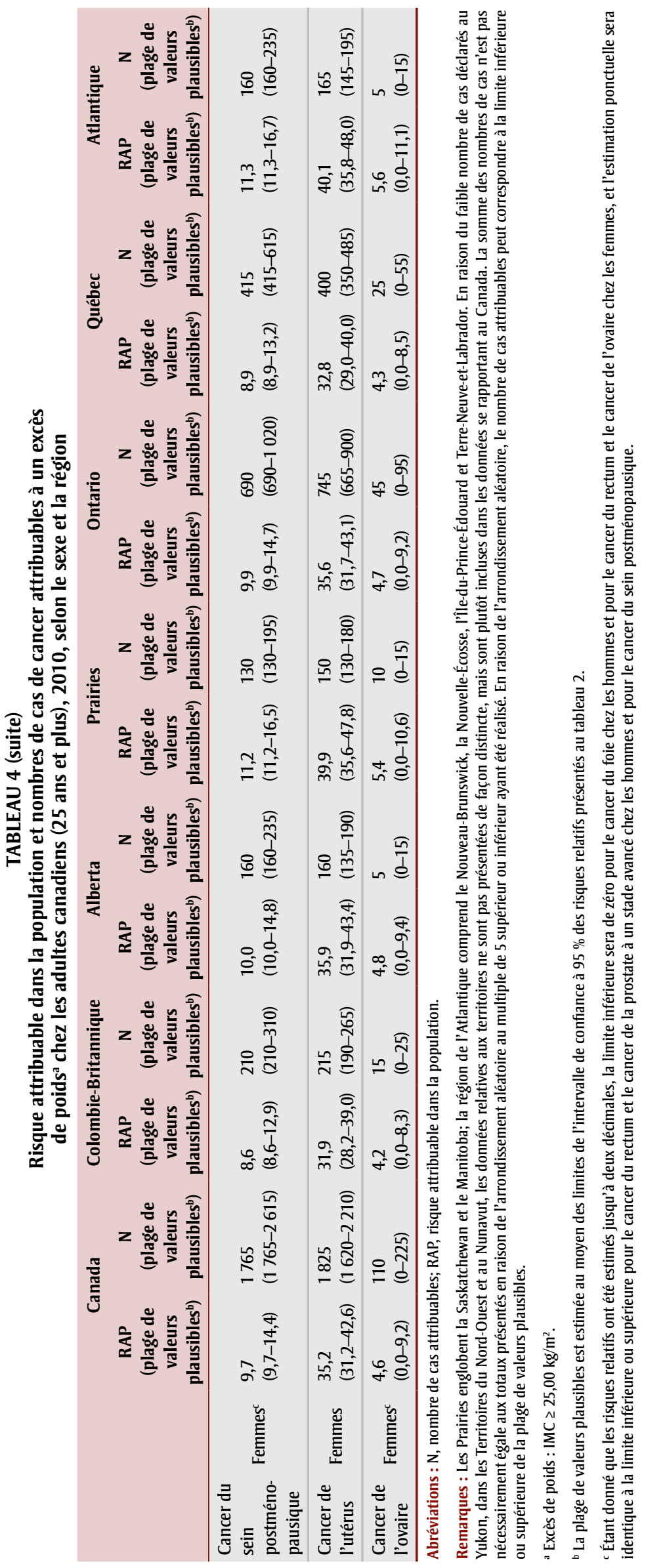

l'ensemble des cas de cancer attribuables à un excès de poids. Chez les femmes, le cancer du sein postménopausique arrive au septième rang du point de vue du RAP, mais au deuxième rang du point de vue du nombre de cas attribuables, ce qui correspond à environ le tiers de l'ensemble des cas de cancer attribuables à un excès de poids.

\section{Analyse}

On peut attribuer environ 5,7 \% des cas de cancer diagnostiqués chez les adultes canadiens en 2010 , soit 1 cas sur 18 , à un excès de poids (IMC $\geq 25,00 \mathrm{~kg} / \mathrm{m}^{2}$ ). Cela correspond à près de 10000 cas de cancer, nombre qui devrait augmenter étant donné la hausse de la prévalence du surpoids et de l'obésité au Canada. Si l'on tient compte de l'incertitude associée à l'ampleur de la relation entre l'excès de poids et le risque de cancer, on obtient un RAP variant entre $4,1 \%$ et $7,6 \%$, et un nombre de cas de cancer attribuables variant entre 6980 et 12845 .

Le RAP varie en fonction du type de cancer, du sexe et de la région. Chez les hommes, les RAP les plus élevés correspondaient à l'adénocarcinome de l'œsophage (42,2\%), au cancer du rein $(25,4 \%)$ et aux cancers du cardia, du foie, du côlon et de la vésicule biliaire (environ 1 sur 5). Chez les femmes, les RAP les plus élevés correspondaient à l'adénocarcinome de l'œsophage $(36,1 \%)$, au cancer de l'utérus $(35,2 \%)$ et aux cancers de la vésicule biliaire et du rein (environ 1 sur 4). De façon générale, les RAP les plus élevés correspondaient aux Prairies (sauf en Alberta) et à la région de l'Atlantique, les plus faibles se situant en Colombie-Britannique et au Québec, suivant la prévalence régionale de l'excès de poids enregistrée en 2000 et en 2001.

Les comparaisons avec les travaux de recherche antérieurs sont complexes, car bien des facteurs influent sur les RAP, en particulier le nombre de cancers dont on tient compte, le RR attribué aux catégories « surpoids » et « obèse » et la prévalence du surpoids et de l'obésité dans la population. Arnold et collab. ${ }^{12}$ ont estimé la proportion des cas de cancer attribuables à un excès de poids en 2012 en utilisant des distributions modélisées de l'IMC, une distribution de type " risque minimum théorique " de l'IMC (moyenne $=22 \mathrm{~kg} / \mathrm{m}^{2}$, écarttype $=1 \mathrm{~kg} / \mathrm{m}^{2}$ ) et des projections du nombre de cas pour chaque type de cancer. 
Chez les hommes, les RAP que nous avons calculés se sont révélés très semblables aux estimations d'Arnold et collab. ${ }^{12}$ pour le Canada. Toutefois, chez les femmes, les RAP présentés dans l'étude d'Arnold et collab. ${ }^{12}$ sont considérablement plus élevés que les nôtres dans le cas de l'adénocarcinome de l'œsophage $(44,0 \%$ contre $36,1 \%)$, du cancer de la vésicule biliaire $(49,0 \%$ contre $23,7 \%)$, du cancer du rein (31,0\% contre $23,0 \%$ ) et du cancer de l'utérus (43,0 \% contre 35,2\%). Certains de ces écarts sont explicables par des différences sur le plan méthodologique, notamment dans la modélisation des données, la formulation des hypothèses sousjacentes et les estimations du RR utilisées. Dans le cas du cancer de la vésicule biliaire et du cancer du rein, Arnold et collab. ${ }^{12}$ ont utilisé les estimations du RR de Renehan et collab., ${ }^{8}$ qui étaient supérieures aux estimations plus récentes mentionnées dans le CUP. L'écart s'est révélé particulièrement important dans le cas de cancer de la vésicule biliaire, soit de 1,59 contre 1,25 pour $5 \mathrm{~kg} / \mathrm{m}^{2}$.

Il existe également des différences entre certains des RAP spécifiques à un cancer et spécifiques à un sexe que nous avons calculés et ceux estimés par Brenner ${ }^{13}$ pour le Canada en 2007. Notre RAP pour l'adénocarcinome de l'œsophage chez les hommes s'est révélé plus élevé $(42,2 \%$ contre $32,3 \%$ ) après divers calculs supplémentaires fondés sur des hypothèses visant à ajuster l'estimation de Brenner pour l'ensemble des cas de cancer de l'œsophage. Notre RAP pour le cancer de la vésicule biliaire est plus élevé à la fois pour les hommes $(20,2 \%$ contre $13,9 \%)$ et pour les femmes $(23,7 \%$ contre $13,0 \%)$. Enfin, nos estimations relatives au cancer du côlon sont considérablement plus élevées que celles de Brenner pour les hommes (20,5\% contre $10,6 \%)$ mais pas pour les femmes $(9,7 \%$ contre $8,9 \%)$.

Outre le fait que notre étude visait une période plus récente, plusieurs facteurs pourraient expliquer les différences observées. En premier lieu, pour le cancer du côlon, nous avons utilisé des estimations plus récentes du $\mathrm{RR}$ spécifique au sexe, et celles-ci se sont révélées plus élevées pour les hommes que pour les femmes. En deuxième lieu, notre méthode consistant à attribuer un RR aux catégories " surpoids » et " obèse " tient compte de la distribution des IMC dans chaque catégorie alors que Brenner ${ }^{13}$ a utilisé des estimations du RR pour une augmentation de
$5 \mathrm{~kg} / \mathrm{m}^{2}$ dans la catégorie « surpoids » et le carré de cette valeur pour la catégorie " obèse ». En troisième lieu, les estimations de Brenner sont fondées sur des IMC non ajustés alors que nous avons ajusté les IMC pour tenir compte du biais associé à l'autodéclaration de la taille et du poids. En dernier lieu, les RAP les plus détaillés de Brenner à partir desquels ont été calculés les autres RAP et les nombres de cas attribuables ne tiennent pas compte de la région alors que les nôtres en tiennent compte.

Enfin, les RAP publiés antérieurement pour l'Ontario en 2010 par Action Cancer Ontario $^{14}$ sont semblables aux nôtres pour le cancer du pancréas $(11,3 \%$ contre $10,4 \%)$, le cancer du rein $(22,8 \%$ contre $24,7 \%)$, le cancer du sein postménopausique $(10,3 \%$, après exclusion des cas de cancer du sein diagnostiqués avant l'âge de 50 ans, contre 9,9\%) et le cancer de l'utérus $(33,0 \%$ contre 35,6\%), mais ils sont différents dans le cas de l'adénocarcinome de l'œsophage $(34,1 \%$, après exclusion des cas ne consistant pas en un adénocarcinome, contre $41,7 \%$ ) et du cancer colorectal $(8,2 \%$ contre $15,3 \%$ pour le cancer du côlon et 8,5\% pour le cancer du rectum). Action Cancer Ontario a corrigé les IMC pour tenir compte du biais associé à l'autodéclaration de données mais d'autres différences méthodologiques demeurent : nous avons utilisé, contrairement à Action Cancer Ontario, des estimations du RR spécifiques au sexe, notamment des estimations distinctes pour le cancer du côlon et le cancer du rectum; nous avons utilisé l'IMC médian d'une catégorie de poids donnée pour lui attribuer un RR, alors qu'Action Cancer Ontario a utilisé une approche semblable à celle de Brenner ${ }^{13}$ et enfin nous avons obtenu les nombres de cas de cancer pour l'Ontario en nous fondant sur une synthèse de la situation fournie par le RCC en septembre 2012 alors qu'Action Cancer Ontario a utilisé les nombres de cas de cancer fournis directement par le Registre des cas de cancer de l'Ontario.

\section{Points forts et limites}

Notre étude présente plusieurs points forts par rapport aux travaux de recherche publiés au Canada jusqu'ici. Premièrement, dans notre étude, nous avons tenu compte des RAP associés à d'autres cancers pour lesquels des données probantes révèlent l'existence d'une relation de cause à effet avec l'excès de poids. Deuxièmement, les RR attribués à la catégorie " surpoids » et à la catégorie " obèse » tiennent compte de la distribution de l'IMC au sein de ces catégories par rapport à un IMC optimal (21 kg/ $\mathrm{m}^{2}$ ). Troisièmement, nous présentons les RAP et les nombres de cas de cancer attribuables à l'excès de poids pour les diverses régions au Canada et pour l'ensemble du pays. Quatrièmement, nous avons corrigé les IMC pour tenir compte du biais lié à l'autodéclaration des données en utilisant des formules conçues et validées en fonction des répondants à l'ESCC. Le fait d'utiliser des IMC corrigés a conduit à une augmentation du RAP global d'environ $20 \%$, le faisant passer de $4,8 \%$ (hommes, 4,2 \%; femmes, 5,4 \%) à $5,7 \%$ (hommes, $4,9 \%$; femmes, 6,5 \%).

Il convient toutefois de mentionner certaines limites pour mieux interpréter les résultats de la présente étude.

En premier lieu, l'IMC ne permet pas de distinguer le poids associé au tissu musculaire de celui associé au tissu adipeux, alors que la relation entre l'IMC et la masse adipeuse varie en fonction de la constitution physique des personnes, de leur sexe, de leur âge et de leur origine ethnique $^{16}$.

En deuxième lieu, le fait de mesurer l'IMC à un moment précis ne tient pas compte des effets, sur le risque de cancer liés à un IMC élevé, spécifiques au type de cancer et dépendants du temps. Selon certains travaux de recherche, le risque de cancer associé à un IMC élevé augmente en fonction du nombre d'années passées avec un IMC élevé, et l'association entre l'IMC et le risque de cancer pourrait se révéler ainsi plus forte chez les jeunes adultes que chez les personnes plus âgées ${ }^{41-43}$.

En troisième lieu, l'association entre l'IMC et le cancer peut différer d'une population à une autre $^{44}$, et les mesures de l'association utilisées dans notre étude ne sont pas spécifiques au Canada. Renehan et collab. ${ }^{8}$ ont cependant constaté que, pour bon nombre de cancers, la hausse du risque associée à une augmentation de l'IMC était cohérente d'une population à une autre. Il convient de mentionner une exception notable, celle du cancer du sein : on a constaté que l'augmentation de l'IMC était associée à une hausse du risque de cancer du sein préménopausique dans les régions de l'Asie-Pacifique, mais à 
une diminution de ce risque dans les autres régions, et le risque de cancer du sein postménopausique associé à une augmentation de l'IMC était plus important dans les régions de l'Asie-Pacifique qu'ailleurs. L'estimation du RR utilisée pour le cancer du sein postménopausique dans notre étude était cohérente avec la valeur applicable pour l’Amérique du Nord.

En quatrième lieu, les RR utilisés dans l'équation 3 ne devraient pas être ajustés pour tenir compte des variables de confusion $^{4}$. Pour que l'équation 3 puisse être utilisée de façon adéquate avec des RR ajustés, il faudrait disposer de données sur l'exposition et la maladie qui soient stratifiées en fonction des variables de confusion, une situation qui ne se présente généralement pas. Fait intéressant, selon Renehan et collab. ${ }^{8}$, la répétition des analyses avec des RR présentant un ajustement minimum plutôt que maximum n'a pas conduit à des résultats différents en ce qui concerne l'association entre l'IMC et le cancer. De plus, le fait de calculer les RAP pour des strates spécifiques à l'âge et spécifiques au sexe, deux variables couramment utilisées pour ajuster les RR, pourrait atténuer l'impact de l'utilisation de RR ajustés dans notre étude ${ }^{44}$.

En cinquième lieu, il existe d'autres types de cancer (méningiome, cancer de la glande thyroïde, myélome multiple) qui n’ont pas été inclus dans notre étude, mais pour lesquels on a récemment déterminé que les données probantes étaient suffisantes pour établir l'existence d'une association avec l'excès de poids. Par conséquent, les RAP et les nombres de cas attribuables mentionnés dans notre étude sont vraisemblablement inférieurs au véritable fardeau du cancer associable à un excès de poids chez les Canadiens d'âge adulte $^{10}$.

En sixième lieu, malgré le fait que le taux de participation à l'ESCC ait été élevé $(84,7 \%)$, les échantillons sont sujets à un biais de non-réponse. Ainsi, si les RAP et les nombres de cas attribuables présentés dans notre étude seraient biaisés à la baisse si les non-participants se révélaient plus susceptibles d'être en surpoids ou obèses que les participants, et inversement.

En septième et dernier lieu, il convient de préciser que les RAP ne sont pas des estimations exactes et exemptes d'erreurs. Au contraire, il existe une incertitude intrinsèque attribuable à la variation des RR ayant été utilisés et à la variation des estimations de la prévalence du surpoids et de l'obésité, ainsi qu'une incertitude statistique inhérente au fait de réaliser des estimations. Afin de tenir compte de cette incertitude, nous avons effectué des estimations de plages de valeurs plausibles pour les RAP et les nombres de cas de cancer attribuables en utilisant les intervalles de confiance à $95 \%$ associés aux RR présentés dans le tableau 2.

\section{Conclusion}

On peut attribuer environ 5,7 \% de l'ensemble des nouveaux cas de cancer diagnostiqués chez les adultes canadiens en 2010, soit 1 cas sur 18, à un IMC élevé, après prise en compte du biais lié à l'autodéclaration de la taille et du poids. Étant donné que la prévalence du surpoids et de l'obésité continue à augmenter au Canada, la proportion de nouveaux cas de cancer attribuables à un excès de poids va également continuer à augmenter à court terme. Il est nécessaire de sensibiliser le public à la relation entre poids et cancer et aux moyens efficaces de maintenir un poids santé. Étant donné l'interrelation entre le poids, le niveau d'activité physique et l'alimentation, les initiatives de santé publique faisant la promotion d'un poids santé par l'activité physique et une alimentation saine auront ainsi vraisemblablement des bienfaits additionnels.

\section{Conflits d'intérêts}

Les auteurs déclarent n'avoir aucun conflit d'intérêts.

\section{Contributions des auteurs}

Les deux auteurs ont contribué à la conception de l'étude, à l'interprétation des résultats, à la rédaction du manuscrit et aux révisions essentielles. D. Z. a effectué les analyses.

\section{Références}

1. Société canadienne du cancer, Comité consultatif des statistiques canadiennes sur le cancer. Statistiques canadiennes sur le cancer 2015. Toronto (Ont.) : Société canadienne du cancer; 2015. 159 p.
2. Statistique Canada. CANSIM, tableau 102-0552. Décès et taux de mortalité (normalisation selon l'âge utilisant la population de 1991), selon certains groupes de causes et le sexe, Canada, provinces et territoires, 2014. [Internet]. Ottawa (Ont.) : Statistique Canada; [consulté le 2 mai 2016]. En ligne à : http://www5.statcan.gc.ca/cansim /a26; jsessionid $=01563127745$ D5A8B3 8A8EDB0D883 AC70? $\mathrm{id}=1020552 \&$ pattern $=\& \mathrm{p} 2=-1 \&$ tabMode $=$ dataTable $\& \mathrm{p} 1=1 \& \mathrm{stByVal}=1 \& \mathrm{csid}=$ \&retrLang $=$ fra\&lang $=$ fra

3. Last JM, editor. A dictionary of epidemiology. $3^{\text {e }}$ éd. New York (NY): Oxford University Press; 1995. 208 p.

4. Rockhill B, Newman B, Weinberg C. Use and misuse of population attributable fractions. Am J Public Health. 1998;88(1):15-9.

5. Parkin DM, Boyd L, Walker LC. 16. The fraction of cancer attributable to lifestyle and environmental factors in the UK in 2010: summary and conclusions. Br J Cancer. 2011; 105 Suppl 2:S77-S81.

6. International Agency for Research on Cancer (IARC), World Health Organization. IARC handbooks of cancer prevention. Volume 6: Weight control and physical activity. Lyon: IARC Press; 2002. 326 p.

7. World Cancer Research Fund, American Institute for Cancer Research (AICR). Food, nutrition, physical activity, and the prevention of cancer: a global perspective. Washington (DC): AICR; 2007. Chapter 2, The cancer process; p. 30-46.

8. Renehan AG, Tyson $M$, Egger $M$, Heller RF, Zwahlen M. Body-mass index and incidence of cancer: a systematic review and meta-analysis of prospective observational studies. Lancet. 2008;371(9612):569-578.

9. Schmid D, Ricci C, Behrens G, Leitzmann MF. Adiposity and risk of thyroid cancer: a systematic review and meta-analysis. Obes Rev. 2015; 16(12):1042-1054.

10. Lauby-Secretan B, Scoccianti C, Loomis $\mathrm{D}$, et al. Body fatness and cancerviewpoint of the IARC Working Group. N Engl J Med. 2016;375(8):794-798. 
11. World Health Organization, International Agency for Research on Cancer. IARC monographs on the evaluation of carcinogenic risks to humans: internal report 14/002. Lyon (FR): IARC Press; 2014. 60 p.

12. Arnold M, Pandeya N, Byrnes G, et al. Global burden of cancer attributable to high body-mass index in 2012: a population-based study. Lancet Oncol. 2015;16(1):36-46.

13. Brenner DR. Cancer incidence due to excess body weight and leisure-time physical inactivity in Canada: implications for prevention. Prev Med. 2014;66:131-139.

14. Cancer Care Ontario. Cancer risk factors in Ontario: healthy weights, healthy eating, and active living. Toronto (Ont.): Queen's Printer for Ontario; 2015. 62 p.

15. Statistique Canada. Embonpoint et obésité chez les adultes (mesures autodéclarées), 2014 [Internet]. Ottawa (Ont.) : Statistique Canada; [consulté le 22 nov. 2015]. En ligne à: http:// www.statcan.gc.ca/pub/82-625-x /2015001/article/14185-fra.htm

16. OMS. Obésité : prévention et prise en charge de l'épidémie mondiale. Genève : Organisation mondiale de la santé; 2003. 284 p.

17. Statistique Canada. Enquête sur la santé dans les collectivités canadiennes (ESCC) [Internet]. Ottawa (Ont.): Statistique Canada; 2001 [modifié le 24 oct. 2007; consulté le 26 nov. 2015]. En ligne à : http://www23. statcan.gc.ca/imdb/p2SV_f.pl?Function $=$ getSurvey\&Id $=3359$

18. Shields M, Gorber SC, Tremblay MS. Estimates of obesity based on selfreport versus direct measures. Health Rep. 2008;19(2):61-76.

19. Gorber SC, Shields M, Tremblay MS, McDowell I. The feasibility of establishing correction factors to adjust self-reported estimates of obesity. Health Rep. 2008;19(3):71-82.

20. Shields M, Gorber SC, Janssen I, Tremblay MS. Bias in self-reported estimates of obesity in Canadian health surveys: an update on correction equations for adults. Health Rep. 2011;22(3):35-45.
21. Kendall BJ, Wilson LF, Olsen CM, et al. Cancers in Australia in 2010 attributable to overweight and obesity. Aust N Z J Public Health. 2015;39(5): 452-457.

22. Parkin DM, Boyd L. 8. Cancers attributable to overweight and obesity in the UK in 2010. Br J Cancer. 2011;105 Suppl 2:S34-S37.

23. World Cancer Research Fund (WCRF) International. Continuous Update Project findings and reports [Internet]. London (UK): WCRF International; 2017 [consulté le 1er déc. 2016]. En ligne à : http://www.wcrf.org/int/research-we -fund/continuous-update-project -findings-reports

24. World Cancer Research Fund (WCRF) International, American Institute for Cancer Research. Continuous Update Project report: Diet, nutrition, physical activity and oesophageal cancer. London (UK): WCRF International; 2016. En ligne à : http://www.wcrf .org/sites/default/files/CUP\% 20 OESOPHAGEAL_WEB.pdf

25. World Cancer Research Fund (WCRF) International, American Institute for Cancer Research. Continuous Update Project report: Diet, nutrition, physical activity and stomach cancer. London (UK): WCRF International; 2016. En ligne à la page : http://wcrf.org/sites /default/files/Stomach-Cancer-2016 -Report.pdf

26. World Cancer Research Fund (WCRF) International, American Institute for Cancer Research. Continuous Update Project report: Diet, nutrition, physical activity and liver cancer. London (UK): WCRF International; 2015. En ligne à : http://wcrf.org/sites/default /files/Liver-Cancer-2015-Report.pdf

27. World Cancer Research Fund (WCRF) International, American Institute for Cancer Research. Continuous Update Project report: Diet, nutrition, physical activity and gallbladder cancer. London (UK): WCRF International; 2015. En ligne à : http://www.wcrf.org/sites /default/files/Gallbladder-Cancer-2015 -Report.pdf
28. World Cancer Research Fund (WCRF), American Institute for Cancer Research. Continuous Update Project report. Food, nutrition, physical activity, and the prevention of pancreatic cancer. London (UK): WCRF; 2012. En ligne à : http://wcrf.org/sites/default/files /Pancreatic-Cancer-2012-Report.pdf

29. World Cancer Research Fund (WCRF), American Institute for Cancer Research. Continuous Update Project report: Food, nutrition, physical activity, and the prevention of colorectal cancer. London (UK): WCRF; 2011. En ligne à : http://wcrf.org/sites/default/files /Colorectal-Cancer-2011-Report.pdf

30. World Cancer Research Fund (WCRF) International, American Institute for Cancer Research. Continuous Update Project report: Diet, nutrition, physical activity and kidney cancer [Internet]. London (UK): WCRF International; 2015. En ligne à : http://wcrf.org/sites/default /files/Kidney-Cancer-2015-Report.pdf

31. World Cancer Research Fund (WCRF) International, American Institute for Cancer Research. Continuous Update Project report: Diet, nutrition, physical activity, and prostate cancer [Internet]. London (UK): WCRF International; 2014. En ligne à : www.wcrf.org/sites/default /files/Prostate-Cancer-2014-Report.pdf

32. World Cancer Research Fund (WCRF), American Institute for Cancer Research. Continuous Update Project report. Food, nutrition, physical activity, and the prevention of breast cancer [Internet]. London (UK): WCRF; 2010 . En ligne à : http://www.wcrf.org/sites/default /files/Breast-Cancer-2010-Report.pdf

33. World Cancer Research Fund (WCRF), American Institute for Cancer Research. Continuous Update Project report. Food, nutrition, physical activity, and the prevention of endometrial cancer [Internet]. London (UK): WCRF; 2013. En ligne à : http://www.wcrf.org/sites/default/files /Endometrial-Cancer-2013-Report.pdf

34. World Cancer Research Fund (WCRF), American Institute for Cancer Research. Continuous Update Project report. Food, nutrition, physical activity, and the prevention of ovarian cancer [Internet]. London (UK): WCRF; 2014. En ligne à : http://www.wcrf.org/sites /default/files/Ovarian-Cancer-2014 -Report.pdf 
35. International Agency for Research on Cancer, World Health Organization, International Association of Cancer Registries, European Network of Cancer Registries. International rules for multiple primary cancers (ICD-O). 3e éd. Lyon : International Agency for Research on Cancer; 2004. [Internal Report $n^{\circ}$ 2004/02]

36. Statistique Canada. CANSIM: Tableau 103-0550: Nouveaux cas de cancer primitif (d'après le fichier des totalisations du RCC de juillet 2016), selon le type de cancer, le groupe d'âge et le sexe, Canada, provinces et territoires [Internet]. Ottawa (Ont.) : Statistique Canada; [consulté le 2 mai 2016]. En ligne à : http://www5.statcan.gc.ca /cansim/a26;jsessionid = E04F124C1D 345A6380ED0BFDCBB46D51?id $=1030550 \&$ pattern $=\& \mathrm{p} 2=-1 \&$ tabMode = dataTable\&p $1=1 \&$ stByVal $=1 \&$ csid

$=$ \&retrLang $=$ fra\&lang $=$ fra3 7

37. Organisation mondiale de la santé. Classification internationale des maladies pour l'oncologie, (CIM-O). $3^{\text {e }}$ éd., $1^{\mathrm{e}}$ révision. Genève : Organisation mondiale de la santé; 2013. 252 p.

38. National Cancer Institute Surveillance, Epidemiology and End Results Program. Site recode ICD-O-3/WHO 2008 definition [Internet]. Bethesda (MD): National Cancer Institute; [consulté le 2 mai 2016]. En ligne à : http://seer.cancer .gov/siterecode/icdo3_dwhoheme /index.html

39. Howlader N, Noone AM, Krapcho M, et al., editors. SEER cancer statistics review, 1975-2013 [Internet]. Bethesda (MD): National Cancer Institute, 2016 [d'après les données soumises au SEER en nov. 2015; consulté le 2 déc. 2016]. En ligne à : http://seer.cancer.gov/csr /1975_2013

40. Philip W, James T, Jackson-Leach R, et al. Overweight and obesity (high body mass index). In: Ezzati M, Lopez AD, Rodgers A, Murray CJL, editors. Comparative quantification of health risks. Geneva $(\mathrm{CH})$ : World Health Organization; 2004:497-596.

41. Abdullah A, Wolfe R, Stoelwinder JU et al. The number of years lived with obesity and the risk of all-cause and cause-specific mortality. Int J Epidemiol. 2011;40(4):985-996.
42. Stolzenberg-Solomon RZ, Schairer C, Moore S, Hollenbeck A, Silverman DT. Lifetime adiposity and risk of pancreatic cancer in the NIH-AARP Diet and Health Study cohort. Am J Clin Nutr. 2013;98(4):1057-1065.

43. Leitzmann MF, Koebnick C, Danforth $\mathrm{KN}$, et al. Body mass index and risk of ovarian cancer. Cancer. 2009;115(4): 812-822.

44. Flegal KM, Panagiotou OA, Graubard BI. Estimating population attributable fractions to quantify the health burden of obesity. Ann Epidemiol. 2015;25(3): 201-207. 